Pacific Journal of Mathematics

A CERTAIN CLASS OF POLYNOMIALS 


\section{A CERTAIN CLASS OF POLYNOMIALS}

\section{RUTH Goodman}

In generalizing Grace's Theorem on apolar polynomials, it was convenient (see reference 1) to use a set of relations among the coefficients of a pair of polynomials which is invariant under nonsingular linear transformations of the polynomials. Other invariant relations among their coefficients will define other classes of pairs of polynomials. The present paper establishes a set of relations which is both necessary and sufficient to guarantee that two polynomials of degree $n$ either have a common zero of multiplicity at least $n-1$ or have their zeros all lying on one circle and so related that if the zeros of one polynomial are transformed, by a linear transformation, into the $n$th roots of +1 then the zeros of the other are carried, by the same transformation, into the $n$th roots of -1 .

A generalization of Grace's theorem [2] on apolar polynomials [1] makes use of a set of relations among the coefficients of a pair of polynomials. An important feature of this set of relations is its invariance under nonsingular linear fractional transformations of the polynomials. Other sets of relations which are similarly invariant will define other classes of polynomials. It is the purpose of this paper to investigate one such class.

For a discussion of Grace's theorem and related results, as well as an extensive bibliography, the reader is referred to Marden's volume [4] in the Mathematical Surveys series.

Let the complex polynomials

$$
\begin{aligned}
& f(z)=\sum_{j=0}^{n}\left(\begin{array}{c}
n \\
j
\end{array}\right) a_{j} z^{j}, \\
& g(z)=\sum_{j=0}^{n}\left(\begin{array}{c}
n \\
j
\end{array}\right) b_{j} z^{j}
\end{aligned}
$$

be given. Application of the linear transformation

$$
z=\frac{\alpha w+\beta}{\gamma w+\delta}
$$

to these polynomials gives the transformed polynomials

$$
F(w)=(\gamma w+\delta)^{n} f\left(\frac{\alpha w+\beta}{\gamma w+\delta}\right)=\sum_{r=0}^{n}\left(\begin{array}{l}
n \\
r
\end{array}\right) A_{r} w^{r},
$$

Received August 8, 1964, and in revised form November 8, 1964. 


$$
G(w)=(\gamma w+\delta)^{n} g\left(\frac{\alpha w+\beta}{\gamma w+\delta}\right)=\sum_{r=0}^{n}\left(\begin{array}{l}
n \\
r
\end{array}\right) B_{r} w^{r} .
$$

LEMMA. The conditions

$$
\begin{gathered}
a_{0} b_{j}+a_{j} b_{0}-a_{s} b_{j-s}-a_{j-s} b_{s}=0 \\
\left(s=1, \cdots,\left[\frac{j}{2}\right] ; j=2, \cdots, n\right), \\
a_{j} b_{n}+a_{n} b_{j}-a_{s} b_{n+j-s}-a_{n+j-s} b_{s}=0 \\
\left(s=j+1, \cdots,\left[\frac{n+j}{2}\right] ; j=1, \cdots, n-2\right)
\end{gathered}
$$

are invariant under nonsingular linear transformations of the polynomials (1).

Proof. Any nonsingular linear transformation is equivalent to a succession of transformations of the types

$$
\begin{array}{ll}
z=\mu w & (\mu \neq 0), \\
z & =\frac{1}{w}, \\
z & =w+\mu .
\end{array}
$$

Hence we can prove that the set of conditions under consideration is invariant under a general nonsingular linear transformation by showing that it is invariant under each of these three types.

Suppose the polynomials (1) satisfy conditions (2a) and (2b).

If $z=\mu w$ is applied to (1), the transformed polynomials have coefficients

$$
\begin{aligned}
& A_{r}=\mu^{r} a_{r}, \\
& B_{r}=\mu^{r} b_{r}
\end{aligned} \quad(r=0, \cdots, n) .
$$

Thus

$$
\begin{array}{r}
A_{0} B_{j}+A_{j} B_{0}-A_{s} B_{j-s}-A_{j-s} B_{s}=\mu^{j}\left(a_{0} b_{j}+a_{j} b_{0}-a_{s} b_{j-s}-a_{j-s} b_{s}\right)=0 \\
\left(s=1, \cdots,\left[\frac{j}{2}\right] ; j=2, \cdots, n\right) .
\end{array}
$$

Similarly

$$
\begin{aligned}
A_{j} B_{n}+A_{n} B_{j} & -A_{s} B_{n+j-s}-A_{n+j-s} B_{s}=\mu^{j+n}\left(a_{j} b_{n}+a_{n} b_{j}\right. \\
- & \left.a_{s} b_{n+j-s}-a_{n+j-s} b_{s}\right)=0 \\
& \left(s=j+1, \cdots,\left[\frac{n+j}{2}\right] ; j=1, \cdots, n-2\right) .
\end{aligned}
$$


When $z=1 / w$, we have

$$
\begin{aligned}
& A_{r}=a_{n-r}, \\
& B_{r}=b_{n-r}
\end{aligned} \quad(r=0, \cdots, n) .
$$

so that the left sides of conditions (2a) for the transformed polynomials are

$$
\begin{aligned}
& A_{0} B_{j}+A_{j} B_{0}-A_{s} B_{j-s}-A_{j-s} B_{s} \\
& =a_{n} b_{n-j}+a_{n-j} b_{n}-a_{n-s} b_{n-j+s}-a_{n-j \div s} b_{n-s} \\
& \quad\left(s=1, \cdots,\left[\frac{j}{2}\right] ; j=2, \cdots, n\right) .
\end{aligned}
$$

With $j^{\prime}=n-j$ and $s^{\prime}=j^{\prime}+s$, these become

$$
\begin{aligned}
& a_{n} b_{j^{\prime}}+a_{j^{\prime}}, b_{n}-a_{n-s^{\prime}+j^{\prime}} b_{s^{\prime}}-a_{s^{\prime}} b_{n-s^{\prime}-j^{\prime}} \\
& \quad\left(s^{\prime}=j^{\prime}+1, \cdots, j^{\prime}+\left[\begin{array}{c}
n-j^{\prime} \\
2
\end{array}\right] ; j^{\prime}=n-2, \cdots, 0\right) .
\end{aligned}
$$

Since $j^{\prime}$ is an integer,

$$
j^{\prime}+\left[\frac{n-j^{\prime}}{2}\right]=\left[j^{\prime}+\frac{n-j^{\prime}}{2}\right]=\left[\frac{j^{\prime}+n}{2}\right] .
$$

Thus the expressions (3) for which $j^{\prime} \neq 0$ are exactly the left sides of equations $(2 \mathrm{~b})$, whence they vanish. Using $j^{\prime}=0$ in (3) gives

$$
a_{n} b_{0}+a_{0} b_{n}-a_{n-s} b_{s}-a_{s} b_{n-s} \quad\left(s=1, \cdots,\left[\frac{n}{2}\right]\right)
$$

and these expressions are the left sides of (2a) with $j=n$, so that they also vanish. Thus conditions (2a) all hold for the transformed polynomials. Now consider conditions (2b). We have

$$
\begin{aligned}
A_{j} B_{n}+A_{n} B_{j}-A_{s} B_{n+j-s}-A_{n+j-s} B_{s} \\
=a_{n-j} b_{0}+a_{0} b_{n-j}-a_{n-s} b_{s-j}-a_{s-j} b_{n-s} \\
\quad\left(s=j+1, \cdots,\left[\frac{n+j}{2}\right] ; j=1, \cdots, n-2\right) .
\end{aligned}
$$

With $j^{\prime}=n-j$ and $s^{\prime}=s-n+j^{\prime}$, these become

$$
\begin{aligned}
& a_{j^{\prime}} b_{0}+a_{0} b_{j^{\prime}}-a_{j^{\prime}-s^{\prime}} b_{s^{\prime}}-a_{s^{\prime}} b_{j^{\prime}-s} \\
& \left(s^{\prime}=1, \cdots,\left[n-\frac{j^{\prime}}{2}\right]-n+j^{\prime} ; j^{\prime}=n-1, \cdots, 2\right) .
\end{aligned}
$$

Since $-n+j^{\prime}$ is an integer, we have

$$
\left[n-\frac{j^{\prime}}{2}\right]-n+j^{\prime}=\left[n-\frac{j^{\prime}}{2}-n+j^{\prime}\right]=\left[\frac{j^{\prime}}{2}\right] .
$$


Thus the expressions (4) are the left sides of equations (2a) with $j \neq n$, and therefore they vanish. We have now seen that the entire set of conditions (2a) and (2b) is invariant under the inversion $z=1 / w$.

We have left to show that the conditions of the lemma are invariant under the translation $z=w+\mu$. Under this transformation, the polynomials (1) are carried [1] into

$$
\begin{aligned}
& F(w)=f(w+\mu)=\sum_{r=0}^{n}\left(\begin{array}{l}
n \\
r
\end{array}\right) A_{r} w^{r}, \\
& G(w)=g(w+\mu)=\sum_{r=0}^{n}\left(\begin{array}{l}
n \\
r
\end{array}\right) B_{r} w^{r},
\end{aligned}
$$

where

$$
\begin{array}{ll}
A_{r}=\sum_{j=r}^{n}\left(\begin{array}{c}
n-r \\
j-r
\end{array}\right) a_{j} \mu^{j-r}, & \\
B_{r}=\sum_{j=r}^{n}\left(\begin{array}{c}
n-r \\
j-r
\end{array}\right) b_{j} \mu^{j-r} & (r=0, \cdots, n) .
\end{array}
$$

Thus we have

$$
\begin{aligned}
A_{r} B_{s}+A_{s} B_{r}=\sum_{j=r}^{n} \sum_{k=s}^{n}\left(\begin{array}{c}
n-r \\
j-r
\end{array}\right)\left(\begin{array}{l}
n-s \\
k-s
\end{array}\right)\left(a_{j} b_{k}+a_{k} b_{j}\right) \mu^{j+k-r-s} & (r=0, \cdots, n ; s=0, \cdots, n) .
\end{aligned}
$$

Equations (2a) and (2b) can clearly be rewritten so that their left sides are all of form

$$
\begin{aligned}
A_{r} B_{s} & +A_{s} B_{r}-A_{r+1} B_{s-1}-A_{s-1} B_{r+1} \\
& =\sum_{j=r}^{n} \sum_{k=s}^{n}\left(\begin{array}{c}
n-r \\
j-r
\end{array}\right)\left(\begin{array}{l}
n-s \\
k-s
\end{array}\right)\left(a_{j} b_{k}+a_{k} b_{j}\right) \mu^{j+k-r-s} \\
& -\sum_{j=r+1}^{n} \sum_{k=s-1}^{n}\left(\begin{array}{c}
n-r-1 \\
j-r-1
\end{array}\right)\left(\begin{array}{l}
n-s+1 \\
k-s+1
\end{array}\right)\left(a_{j} b_{k}+a_{k} b_{j}\right) \mu^{j+k-r-s} .
\end{aligned}
$$

Using the well known fact that the binomial coefficients satisfy the relations

$$
\begin{aligned}
\left(\begin{array}{l}
n \\
0
\end{array}\right) & =\left(\begin{array}{l}
n \\
n
\end{array}\right)=1, \\
\left(\begin{array}{l}
n \\
h
\end{array}\right) & =\left(\begin{array}{l}
n-1 \\
h-1
\end{array}\right)+\left(\begin{array}{c}
n-1 \\
h
\end{array}\right) \quad(h=1, \cdots, n-1),
\end{aligned}
$$

we can write this last expression as follows:

$$
\sum_{j=r}^{n-1} \sum_{k=s}^{n}\left(\begin{array}{c}
n-r-1 \\
j-r
\end{array}\right)\left(\begin{array}{c}
n-s \\
k-s
\end{array}\right)\left(a_{j} b_{k}+a_{k} b_{j}-a_{j+1} b_{k-1}-a_{k-1} b_{j+1}\right) \mu^{j+k-r-s} .
$$


Thus the left side of every equation (2a) and (2b) for the transformed polynomials is a linear combination of the left sides of the original equations (2a) and (2b). Hence they all vanish and the conditions are invariant under translations.

THEOREM. A necessary and sufficient condition that there exists a nonsingular linear transformation which carries the polynomials (1), with $n \geqq 2$, either into the pair

$$
\begin{aligned}
& c_{1}\left(w^{n}+1\right), \\
& c_{2}\left(w^{n}-1\right)
\end{aligned}
$$

or into the pair

$$
\begin{aligned}
& c_{1} w^{n}, \\
& c_{2}\left(w^{n}+B w^{n-1}\right),
\end{aligned}
$$

where $c_{1} \neq 0, c_{2} \neq 0$ and $B$ are constants, is that the coefficients of (1) satisfy conditions (2a) and (2b).

Proof. Since changing a polynomial by a multiplicative constant does not affect its zeros, we may, without loss of generality, assume $c_{1}=c_{2}=1$.

To prove the necessity, let us assume first that (1) are transformable into $w^{n}+1$ and $w^{n}-1$. Since (2a) and $(2 b)$ are invariant under linear transformations, it is sufficient to check that they hold for the transformed polynomials. Since $A_{n}=B_{n}=1, A_{0}=1$ and $B_{0}=-1$ are the only nonvanishing coefficients, the only equations among (2a) and (2b) which have nonvanishing terms are

$$
A_{0} B_{n}+A_{n} B_{0}-A_{s} B_{n-s}-A_{n-s} B_{s}=A_{0} B_{n}+A_{n} B_{0}=0 .
$$

If (1) are transformable into $w^{n}$ and $w^{n}+B w^{n-1}$, the only nonvanishing coefficients are $A_{n}=B_{n}=1$ and $B_{n-1}=B / n$. Since the product $A_{n} B_{n-1}$ is the only nonvanishing $A_{j} B_{k}$ and since it does not occur in any of the equations (2a) or (2b), all the equations are satisfied.

The proof of the sufficiency will be by mathematical induction.

Let $n=2$ and suppose one of the polynomials, say $f(z)$, has distinct zeros. These can be transformed into the points $w=1$ and $w=-1$, so that $f(z)$ is carried into

$$
F(w)=w^{2}-1,
$$

with $A_{2}=1, A_{1}=0, A_{0}=-1$. If $g(z)$ has a zero distinct from those of $f(z)$, the same transformation can carry this zero into $w=i$, so that $g(z)$ is transformed into 


$$
G(w)=B_{2} w^{2}+2 B_{1} w+B_{0},
$$

where

$$
G(i)=-B_{2}+2 B_{1} i+B_{0}=0 .
$$

The coefficients of $F(w)$ and $G(w)$ now satisfy

$$
0=A_{0} B_{2}+A_{2} B_{0}-2 A_{1} B_{1}=-2 B_{1} i \text {. }
$$

Thus

$$
\begin{aligned}
B_{1} & =0, \\
B_{0} & =B_{2}, \\
G(w) & =B_{2}\left(w^{2}+1\right),
\end{aligned}
$$

as we want. Suppose now that $f(z)$ has distinct zeros, but that both zeros of $g(z)$ are also zeros of $f(z)$. We can transform the zeros of $f(z)$ into $w=0$ and $w=-1$, at the same time taking one zeros of $g(z)$ into $w=0$. Then

$$
\begin{aligned}
& F(w)=w^{2}+w, \\
& G(w)=B_{2} w^{2}+2 B_{1} w,
\end{aligned}
$$

so that

$$
0=A_{0} B_{2}+A_{2} B_{0}-2 A_{1} B_{1}=-2 B_{1} .
$$

Thus $B_{1}=0$ and $F(w)$ and $G(w)$ are of one of the specified forms. If neither polynomial has distinct zeros, we can transform $f(z)$ into

$$
F(w)=w^{2} \text {. }
$$

We have

$$
G(w)=B_{2} w^{2}+2 B_{1} w+B_{0},
$$

with coefficients which satisfy

$$
0=A_{0} B_{2}+A_{2} B_{0}-2 A_{1} B_{1}=B_{0} \text {. }
$$

Thus

$$
G(w)=B_{2} w^{2}+2 B_{1} w,
$$

and $F(w)$ and $G(w)$ are again of one of the specified forms.

Now assume that $f(z)$ and $g(z)$ are of degree $n$ and that the theorem is known to hold for polynomials of degree $n-1$. The derivatives

$$
f^{\prime}(z)=\sum_{j=1}^{n}\left(\begin{array}{c}
n \\
j
\end{array}\right) j a_{j} z^{j-1}=n \sum_{j^{\prime}=0}^{n-1}\left(\begin{array}{c}
n-1 \\
j^{\prime}
\end{array}\right) a_{j^{\prime}+1} z^{j^{\prime}},
$$




$$
g^{\prime}(z)=\sum_{j=1}^{n}\left(\begin{array}{l}
n \\
j
\end{array}\right) j b_{j} z^{j-1}=n \sum_{j^{\prime}=0}^{n-1}\left(\begin{array}{c}
n-1 \\
j^{\prime}
\end{array}\right) b_{j^{\prime}+1} z^{j^{\prime}}
$$

are $(n-1)$ st degree polynomials which satisfy conditions $(2 a)$ and $(2 b)$. Thus $f^{\prime}(z)$ and $g^{\prime}(z)$ are the transforms of either

$$
\begin{aligned}
& F(w)=w^{n-1}-1, \\
& G(w)=w^{n-1}+1
\end{aligned}
$$

or

$$
\begin{aligned}
& F(w)=w^{n-1} \\
& G(w)=w^{n-1}+B w^{n-2}
\end{aligned}
$$

under a linear transformation

$$
w=\frac{\alpha z+\beta}{\gamma z+\delta}
$$

Suppose $f^{\prime}(z)$ and $g^{\prime}(z)$ are the transforms of (5). Then

$$
\begin{aligned}
& f^{\prime}(z)=(\gamma z+\delta)^{n-1} F\left(\frac{\alpha z+\beta}{\gamma z+\delta}\right)=(\alpha z+\beta)^{n-1}-(\gamma z+\delta)^{n-1}, \\
& g^{\prime}(z)=(\gamma z+\delta)^{n-1} G\left(\frac{\alpha z+\beta}{\gamma z+\delta}\right)=(\alpha z+\beta)^{n-1}+(\gamma z+\delta)^{n-1}
\end{aligned}
$$

Since $\beta \gamma-\alpha \hat{\delta} \neq 0$, the coefficients $\alpha$ and $\gamma$ cannot vanish simultaneously. Suppose $\gamma=0$. Then

$$
\begin{aligned}
f^{\prime}(z) & =(\alpha z+\beta)^{n-1}-\hat{o}^{n-1}, \\
g^{\prime}(z) & =(\alpha z+\beta)^{n-1}+\hat{o}^{n-1}, \\
f(z) & =\frac{1}{n \alpha}(\alpha z+\beta)^{n}-\hat{o}^{n-1} z+h, \\
g(z) & =\frac{1}{n \alpha}(\alpha z+\beta)^{n}+\hat{o}^{n-1} z+k,
\end{aligned}
$$

where $h$ and $k$ are constants of integration. The coefficients of $f(z)$ and $g(z)$ are

$$
\begin{aligned}
& a_{0}=\frac{\beta^{n}}{n \alpha}+h, \\
& b_{0}=\frac{\beta^{n}}{n \alpha}+k, \\
& a_{1}=\frac{\beta^{n-1}}{n}-\frac{\delta^{n-1}}{n}, \\
& b_{1}=\frac{\beta^{n-1}}{n}+\frac{\delta^{n-1}}{n},
\end{aligned}
$$




$$
a_{j}=b_{j}=\frac{\alpha^{j-1} \beta^{n-j}}{n}
$$

$$
(j=2, \cdots, n)
$$

Equations (2a) and (2b) now give

$$
0=a_{0} b_{n}+a_{n} b_{0}-a_{1} b_{n-1}-a_{n-1} b_{1}=\frac{\alpha^{n-1}}{n}(h+k) \text {. }
$$

Since $\alpha \neq 0$, we must have $k=-h$. We also have

$$
0=a_{0} b_{2}+a_{2} b_{0}-2 a_{1} b_{1}=\frac{2 \delta^{2 n-2}}{n^{2}}
$$

Thus we have $\delta=0$, so that $\gamma$ and $\delta$ vanish simultaneously. Since $\beta \gamma-\alpha \delta \neq 0$, this cannot happen. Hence $\gamma \neq 0$. If $\alpha=0, \gamma \neq 0$, we can proceed similarly:

$$
\begin{aligned}
f^{\prime}(z) & =\beta^{n-1}-(\gamma z+\delta)^{n-1}, \\
g^{\prime}(z) & =\beta^{n-1}+(\gamma z+\delta)^{n-1}, \\
f(z) & =h+\beta^{n-1} z-\frac{1}{\gamma n}(\gamma z+\delta)^{n}, \\
g(z) & =k+\beta^{n-1} z+\frac{1}{\gamma n}(\gamma z+\delta)^{n}, \\
a_{0} & =h-\frac{\delta^{n}}{n \gamma}, \\
b_{0} & =k+\frac{\delta^{n}}{n \gamma}, \\
a_{1} & =\frac{\beta^{n-1}}{n}-\frac{\delta^{n-1}}{n}, \\
b_{1} & =\frac{\beta^{n-1}}{n}+\frac{\delta^{n-1}}{n}, \\
a_{j} & =-\frac{\gamma^{j-1} \delta^{n-j}}{n} \\
b_{j} & =-a_{j} \\
0 & =a_{0} b_{n}+a_{n} b_{0}-a_{1} b_{n-1}-a_{n-1} b_{1}=\frac{\gamma^{n-1}}{n}(k-h), \\
h & =k, \quad(j=2, \cdots, n) . \\
0 & =a_{0} b_{2}+a_{2} b_{0}-2 a_{1} b_{1}=-\frac{2 \beta^{2 n-2}}{n^{2}}, \quad(j=2, \cdots, n),
\end{aligned}, \quad,
$$

Again, since $\beta \gamma-\alpha \delta \neq 0, \alpha$ and $\beta$ cannot vanish simultaneously. Thus $\alpha \neq 0$. Now we have $\alpha \neq 0$ and $\gamma \neq 0$, and integration of (7) gives 
( 8 )

$$
\begin{aligned}
& f(z)=\frac{1}{n \alpha}(\alpha z+\beta)^{n}-\frac{1}{n \gamma}(\gamma z+\delta)^{n}+h, \\
& g(z)=\frac{1}{n \alpha}(\alpha z+\beta)^{n}+\frac{1}{n \gamma}(\gamma z+\delta)^{n}+k .
\end{aligned}
$$

Thus

$$
\begin{aligned}
& f(z)=\frac{1}{n} \sum_{j=0}^{n}\left(\begin{array}{c}
n \\
j
\end{array}\right)\left(\alpha^{j-1} \beta^{n-j}-\gamma^{j-1} \delta^{n-j}\right) z^{j}+h, \\
& g(z)=\frac{1}{n} \sum_{j=0}^{n}\left(\begin{array}{l}
n \\
j
\end{array}\right)\left(\alpha^{j-1} \beta^{n-j}+\gamma^{j-1} \delta^{n-j}\right) z^{j}+k,
\end{aligned}
$$

and the coefficients of these polynomials are

$$
\begin{aligned}
& a_{0}=p_{0}+h, \\
& b_{0}=q_{0}+k, \\
& a_{j}=p_{j}, \\
& b_{j}=q_{j}
\end{aligned}
$$

$$
(j=1, \cdots, n),
$$

where

$$
\begin{aligned}
& p_{j}=\frac{1}{n}\left(\alpha^{j-1} \beta^{n-j}-\gamma^{j-1} \delta^{n-j}\right), \\
& q_{j}=\frac{1}{n}\left(\alpha^{j-1} \beta^{n-j}+\gamma^{j-1} \delta^{n-j}\right) \quad(j=0, \cdots, n-1), \\
& p_{n}=\frac{1}{n}\left(\alpha^{n-1}-\gamma^{n-1}\right), \\
& q_{n}=\frac{1}{n}\left(\alpha^{n-1}+\gamma^{n-1}\right) .
\end{aligned}
$$

(Note that $p_{n}$ and $q_{n}$ need to be written separately only if $\beta=0$ or $\delta=0$.) Now we observe that

$$
p_{j} q_{k}+p_{k} q_{j}=\frac{2}{n^{2}}\left(\alpha^{j+k-2} \beta^{2 n-j-k}-\gamma^{j+k-2} \delta^{2 n-j-k}\right),
$$

so that the value of $p_{j} q_{k}+p_{k} q_{j}$ depends on the sum $j+k$ but not on $j$ and $k$ individually. Thus we can write

$$
\begin{aligned}
& 0=a_{0} b_{2}+a_{2} b_{0}-2 a_{1} b_{1}=h q_{2}+k p_{2}, \\
& 0=a_{0} b_{3}+a_{3} b_{0}-a_{1} b_{2}-a_{2} b_{1}=h q_{2}+k p_{3} .
\end{aligned}
$$

We now have two simultaneous linear homogeneous equations in $h$ and $k$. For them to have any solution other than $h=k=0$, their determinant must vanish. This determinant is 


$$
p_{3} q_{2}-p_{2} q_{3}=-\frac{2}{n^{2}} \alpha \gamma \beta^{n-3} \delta^{n-3} .
$$

Since $\alpha \gamma \neq 0$, this determinant can vanish only if $\beta=0$ or $\delta=0$. Note that $\beta=\delta=0$ is impossible since $\alpha \hat{\delta}-\beta \gamma \neq 0$. Suppose $\delta=0$. Then $p_{j}=q_{j} \neq 0$ for all $j=0, \cdots, n-1$. Thus from the equation

$$
0=h q_{2}+k p_{2}=(h+k) p_{2}
$$

we find that $h+k=0$, that is, $k=-h$. We now have

$$
0=a_{0} b_{n}+a_{n} b_{0}-a_{1} b_{n-1}-a_{n-1} b_{1}=\frac{2 h}{n} \gamma^{n-1} \text {. }
$$

Thus we must have $h=k=0$. Assume now that the determinant vanishes because $\beta=0$. We have $p_{j}=-q_{j} \neq 0$ for $j=0, \cdots, n-1$. Thus from

$$
0=h q_{2}+k p_{2}=(h-k) q_{2}
$$

we find that $h=k$. Now

$$
0=a_{0} b_{n}+a_{n} b_{0}-a_{1} b_{n-1}-a_{n-1} b_{1}=\frac{2 h}{n} \alpha^{n-1} .
$$

Again we must have $h=0$. That is, the assumption that the determinant (9) vanishes leads to the conclusion that $h=k=0$. Thus whenever $\alpha \gamma \neq 0$, we must have $h=k=0$. Using these values and applying to the polynomials (8) the transformation

$$
z=\frac{\beta-\delta \mu w}{\gamma \mu w-\alpha} \quad(\beta \gamma-\alpha \gamma=1),
$$

where

$$
\mu=\left(\frac{\alpha}{\gamma}\right)^{\frac{1}{n}}
$$

gives

$$
F(w)=(\gamma \mu w-\alpha)^{n} f\left(\frac{\beta-\delta \mu w}{\gamma \mu w-\alpha}\right)=\frac{1}{n \gamma}\left(w^{n}-1\right)
$$

and, similarly,

$$
G(w)=\frac{1}{n \gamma}\left(w^{n}+1\right) .
$$

We have left to deal with the case where $f^{\prime}(z)$ and $g^{\prime}(z)$ are transforms of (6). Suppose they are obtained from (6) by the transformation 


$$
w=\frac{\alpha z+\beta}{\gamma z+\hat{\partial}}
$$

In this event

$$
\begin{aligned}
& f^{\prime}(z)=(\alpha z+\beta)^{n-1}, \\
& g^{\prime}(z)=(\alpha z+\beta)^{n-1}+B(\alpha z+\beta)^{n-2}(\gamma z+\delta) .
\end{aligned}
$$

Now we can assume, without loss of generality, that all the zeros of both $f(z)$ and $g(z)$ are finite. For, if they are not, we can perform a linear transformation which will make them all finite and which will preserve conditions (2a) and (2b). Thus in the above polynomials we can assume $\alpha \neq 0$; for $\alpha=0$ would give $f^{\prime}(z)=\beta^{n-1}, f(z)=\beta^{n-1} z+h$, so that $f(z)$ has $n-1$ zeros at infinity. Integration by parts gives

$$
\int(\alpha z+\beta)^{n-2}(\gamma z+\delta) d z=(\gamma z+\hat{o}) \frac{(\alpha z+\beta)^{n-1}}{\alpha(n-1)}-\frac{\gamma(\alpha z+\beta)^{n}}{\alpha^{2}(n-1) n}+C,
$$

so that

$$
\begin{aligned}
f(z)= & \frac{1}{\alpha n}(\alpha z+\beta)^{n}+h, \\
g(z)= & \frac{1}{\alpha n}\left(1-\frac{B \gamma}{\alpha(n-1)}\right)(\alpha z+\beta)^{n} \\
& +\frac{B}{\alpha(n-1)}(\alpha z+\beta)^{n-1}(\gamma z+\delta)+k .
\end{aligned}
$$

By assumption $f(z)$ and $g(z)$ satisfy conditions (2a) and (2b). Applying the transformation

$$
z=\frac{\beta-\delta w}{\gamma w-\alpha} \quad(\beta \gamma-\alpha \delta=1),
$$

we have

$$
\begin{aligned}
\alpha z+\beta & =\frac{w}{\gamma w-\alpha}, \\
\gamma z+\delta & =\frac{1}{\gamma w-\alpha},
\end{aligned}
$$

so that $f(z)$ and $g(z)$ have transforms

$$
\begin{aligned}
& F(w)=\frac{1}{\alpha n} w^{n}+h(\gamma w-\alpha)^{n}, \\
& G(w)=\frac{1}{\alpha n}\left(1-\frac{B \gamma}{\alpha(n-1)}\right) w^{n}+\frac{B}{\alpha(n-1)} w^{n-1}+k(\gamma w-\alpha)^{n}
\end{aligned}
$$

which also satisfy conditions (2a) and (2b). These polynomials have coefficients 


$$
\begin{array}{rlrl}
a_{n} & =\frac{1}{\alpha n}+h p_{n}, & \\
a_{j} & =h p_{j} & & (j=0, \cdots, n-1), \\
b_{n} & =\frac{1}{\alpha n}\left(1-\frac{B \gamma}{\alpha(n-1)}\right)+k p_{n}, & \\
b_{n-1} & =\frac{B}{\alpha(n-1) n}+k p_{n-1}, & \\
b_{j} & =k p_{j} & (j=0, \cdots, n-2),
\end{array}
$$

where

$$
p_{j}=(-1)^{n-j} \gamma^{j} \alpha^{n-j}
$$$$
(j=0, \cdots, n)
$$

Now

$$
p_{j} p_{k}=(-1)^{j+k} \gamma^{j+k} \alpha^{2 n-j-k}
$$

and this quantity depends only on $j+k$, not on $j$ and $k$ individually. We have

$$
\begin{aligned}
0 & =a_{0} b_{n}+a_{n} b_{0}-a_{1} b_{n-1}-a_{n-1} b_{1} \\
& =\frac{1}{\alpha n}\left\{h p_{0}\left(1-\frac{B \gamma}{\alpha(n-1)}\right)+k p_{0}-h p_{1} \frac{B}{n-1}\right\} .
\end{aligned}
$$

Since $1 / \alpha n \neq 0$, the second factor must vanish. Thus

$$
0=(-1)^{n} \alpha^{n}(h+k)
$$

so that $k=-h$. We also have

$$
0=a_{0} b_{n-1}+a_{n-1} b_{0}-a_{1} b_{n-2}-a_{n-2} b_{1}=\frac{h p_{0} B}{\alpha(n-1) n} .
$$

Since $p_{0}=(-1)^{n} \alpha^{n} \neq 0$, we must now have either $B=0$ or $h=0$. If $h=0$, then $k=-h=0$, and $F(w)$ and $G(w)$ are of the desired form at once. Using $B=0$ and $k=-h$, we have

$$
\begin{aligned}
& F(w)=\frac{1}{\alpha n} w^{n}+h(\gamma w-\alpha)^{n}, \\
& G(w)=\frac{1}{\alpha n} w^{n}-h(\gamma w-\alpha)^{n} .
\end{aligned}
$$

If we now perform the transformation

$$
w=\frac{\alpha \mu u}{\gamma \mu u-1}
$$

where 


$$
\mu=(h \alpha n)^{\frac{1}{n}}
$$

we have

$$
\begin{aligned}
F(w) & =F_{1}(u)=\frac{\alpha^{n-1} \mu^{n}}{n} u^{n}+h \alpha^{n} \\
& =h \alpha^{n}\left(u^{n}+1\right)
\end{aligned}
$$

and, similarly,

$$
G(w)=G_{1}(u)=h \alpha^{n}\left(u^{n}-1\right)
$$

These polynomials are of one of the desired forms, and the proof of the theorem is complete.

The results we have just obtained can be stated in terms of the geometry of the zeros of the two polynomials. When a linear transformation is applied to a polynomial, the zeros of the transform are the transforms [3] of the zeros of the original polynomial. Hence we can state: Conditions $(2 \mathrm{a})$ and $(2 \mathrm{~b})$ are both necessary and sufficient to guarantee that either

(i) $f(z)$ and $g(z)$ have a common, repeated zero of multiplicity at least $n-1$ or

(ii) their zeros all lie on the same circle or straight line and the zeros of the two polynomials separate each other in such a way that a linear transformation will, at the same time, carry those of one polynomial into the $n$th roots of unity and those of the other into the $n$th roots of negative unity.

\section{REFERENCES}

1. R. Goodman, K-polar polynomials, Pacific J. Math. 12 (1962), 1277-1288.

2. J. H. Grace, The zeros of a polynomial, Proc. Cambridge Philos. Soc. 11 (1902), 352-357.

3. E. Laguerre, Oeuvres, Paris: Gauthier-Villars, 1 (1898), 48-66.

4. M. Marden, The geometry of the zeros of a polynomial in a complex variable, Amer. Math. Soc. Math. Surveys No. III, New York (1946), 44-66.

Westinghouse EleCtRIC CORPORATION AND

Rose Polytechnic Institute 



\section{PACIFIC JOURNAL OF MATHEMATICS}

\section{EDITORS}

\section{H. SAMELSON}

Stanford University

Stanford, California

R. M. Blumenthal

University of Washington

Seattle, Washington 98105

\author{
*J. DugundJI \\ University of Southern California \\ Los Angeles, California 90007 \\ RICHARD ARENS \\ University of California \\ Los Angeles, California 90024
}

\section{ASSOCIATE EDITORS}
E. F. BECKENBACH
B. H. NeUManN
F. WolF
K. YosIDA

\section{SUPPORTING INSTITUTIONS}

UNIVERSITY OF BRITISH COLUMBIA
CALIFORNIA INSTITUTE OF TECHNOLOGY
UNIVERSITY OF CALIFORNIA
MONTANA STATE UNIVERSITY
UNIVERSITY OF NEVADA
NEW MEXICO STATE UNIVERSITY
OREGON STATE UNIVERSITY
UNIVERSITY OF OREGON
OSAKA UNIVERSITY
UNIVERSITY OF SOUTHERN CALIFORNIA

UNIVERSITY OF BRITISH COLUMBIA

UNIVERSITY OF CALIFORNIA

MONTANA STATE UNIVERSITY

NEW MEXICO STATE UNIVERSITY

OREGON STATE UNIVERSITY

OSAKA UNIVERSITY

UNIVERSITY OF SOUTHERN CALIFORNIA

\author{
STANFORD UNIVERSITY \\ UNIVERSITY OF TOKYO \\ UNIVERSITY OF UTAH \\ WASHINGTON STATE UNIVERSITY \\ UNIVERSITY OF WASHINGTON \\ AMERICAN MATHEMATICAL SOCIETY \\ CHEVRON RESEARCH CORPORATION \\ TRW SYSTEMS \\ NAVAL ORDNANCE TEST STATION
}




\section{Pacific Journal of Mathematics \\ Vol. 17, No. $1 \quad$ January, 1966}

Carlos Jorge Do Rego Borges, On stratifiable spaces ................ 1

Felix Earl Browder, Topological methods for non-linear elliptic equations of

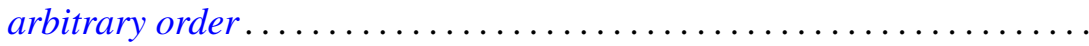

Gustave Choquet, Harry Corson and Victor Klee, Exposed points of convex

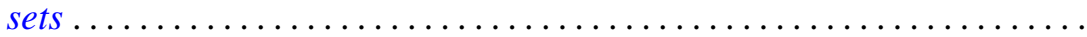

Phillip Emig, Remarks on the defect sum for a function meromorphic on an open Riemann surface ................................ 45

Ruth Goodman, A certain class of polynomials .................. 57

Sidney (Denny) L. Gulick, The bidual of a locally multiplicatively-convex

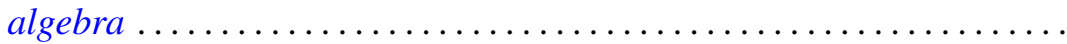

Eugene Carlyle Johnsen, Integral solutions to the incidence equation for finite projective plane cases of orders $n \equiv=2(\bmod 4) \ldots \ldots \ldots \ldots . .67$

Charles N. Kellogg, Centralizers and $H^{*}$-algebras .................. 121

Michael Lodato, On topologically induced generalized proximity relations. II .......................................... 131

P. H. Maserick, Half rings in linear spaces ..................... 137

Kathleen B O'Keefe, On a problem of J. F. Ritt .................... 149

Galen Lathrop Seever, Nonnegative projections on $C_{0}(X) \ldots \ldots \ldots \ldots$

Lawrence A. Shepp, Gaussian measures in function space ............ 167

Robert Charles Thompson, Classes of definite group matrices ........... 175 